\title{
EchoGéo
}

$30 \mid 2014$

Varia

\section{L'accession à la propriété privée dans les villes polonaises : un défi de transition post-socialiste}

Caroline Bouloc and Magdalena Górczyńska

\section{OpenEdition}

12 Journals

Electronic version

URL: https://journals.openedition.org/echogeo/14057

DOI: 10.4000/echogeo.14057

ISSN: 1963-1197

Publisher

Pôle de recherche pour l'organisation et la diffusion de l'information géographique (CNRS UMR 8586)

Electronic reference

Caroline Bouloc and Magdalena Górczyńska, "L'accession à la propriété privée dans les villes polonaises : un défi de transition post-socialiste", EchoGéo [Online], 30 | 2014, Online since 11

December 2014, connection on 31 July 2021. URL: http://journals.openedition.org/echogeo/14057 ; DOI: https://doi.org/10.4000/echogeo.14057

This text was automatically generated on 31 July 2021.

EchoGéo est mis à disposition selon les termes de la licence Creative Commons Attribution - Pas d'Utilisation Commerciale - Pas de Modification 4.0 International (CC BY-NC-ND) 


\title{
L'accession à la propriété privée dans les villes polonaises : un défi de transition post-socialiste
}

\author{
Caroline Bouloc and Magdalena Górczyńska
}

\section{Introduction}

Durant la crise économique de 2009, la Pologne est apparue comme le seul pays européen à avoir maintenu une croissance économique. Toutefois, malgré cette dynamique générale, le pays est confronté à un certain nombre de difficultés issues du passage d'un régime socialiste à un régime capitaliste devenant un espace de l'entredeux ou plus précisément de l'intermédiarité (Rey, 1990 ; Carter, Jordan, Rey, 1998 ; Le Gall, Rougé, 2014). Le secteur résidentiel représente parfaitement cette complexité. D'un côté, les difficultés d'accès aux logements des Polonais deviennent similaires à celles des autres pays d'Europe notamment à cause de l'augmentation accélérée des prix moyens au mètre carré des logements sur le marché immobilier depuis 2008. D'un autre côté, de nombreuses particularités structurelles persistent dans le secteur résidentiel, issues des changements de régimes politiques durant le $\mathrm{XX}^{\mathrm{e}}$ siècle et du manque de logements qui s'est aggravé depuis les années 1980.

2 Le secteur résidentiel polonais a en effet été maintes fois remanié, le plus gros bouleversement ayant sans nul doute été la nationalisation des biens privés après la Seconde Guerre mondiale. L'année 1989 marque la fin de la parenthèse socialiste et permet que la propriété privée redevienne le centre du secteur résidentiel dans un nouveau contexte capitaliste. Le désir de devenir propriétaire s'y impose plus fortement que dans les pays occidentaux, parce que la propriété privée se présente comme l'opposé de l'habitat socialiste (Bouloc, 2013). En effet, la privatisation des biens n'a pas supprimé les lois et les règles socialistes de manière radicale pour embrasser celles du capitalisme occidental (Marcuse, 1996; Zborowski, 2005). Le processus de changement des droits de propriété s'est imposé non pas comme une simple 
redéfinition de son sens et de ses fonctions, mais également comme une politique de redistribution des droits de propriété (Marcuse, 1996 ; Hegedüs, Tosics, 1998 ; Glock, 2007).

3 Comment passe-t-on alors d'un secteur de l'habitat principalement public dans les villes polonaises à une prédominance de la propriété privée ? L'accession à la propriété pour plus de la moitié des citadins polonais en 2011 sonne-t-elle le glas de la transition du secteur de l'habitat? Cette accession a en effet représenté une des difficultés de la politique de la privatisation depuis 1989. Chaque segment, chaque aspect du secteur de l'habitat a été repensé provoquant de nombreux problèmes socio-économiques, mais aussi politiques et créant de nombreuses contradictions qui rendent la lecture de ce secteur plus ardue. Devenir propriétaire de son logement après 1989 passe par différents processus selon le statut d'origine des logements occupés, mais est également lié à la construction immobilière amorcée à la fin des années 1990 dont la majorité des logements est livrée par le secteur privé.

4 Dans le but de répondre à ces questionnements et afin de rendre plus lisibles ces changements, cette étude s'appuie sur les données statistiques de l'Institut général de statistiques polonais (GUS) croisant les données annuelles du secteur de l'habitat et les différents recensements nationaux de la population et des logements $(1988,2002$ et 2011). Cette analyse est complétée par l'étude approfondie de la législation et des nouvelles lois qui ont été établies avant et après 1989 concernant la restructuration du secteur de l'habitat.

5 Ainsi, d'une part, il est nécessaire de revenir sur les bouleversements que le régime socialiste a engendrés sur le principe de propriété privée en Pologne après la Seconde Guerre mondiale. L'année 1989 marquant la fin de cette idéologie sur le secteur de l'habitat en Pologne, nous effectuerons, d'autre part, une présentation des changements survenus dans le secteur de l'habitat s'ouvrant à la propriété privée et la re-création du marché de l'immobilier à partir de cette date. Ensuite, nous présenterons les différents modes d'accession à la propriété dans le parc préexistant et les politiques de l'habitat qui les ont rendus possibles. Enfin, nous évaluerons les conséquences sociales et spatiales que ces nouvelles accessions provoquent en suivant l'exemple de Varsovie.

\section{Remise en question du principe de propriété privée par l'idéologie socialiste jusqu'en 1989}

6 Le secteur de l'habitat en Pologne tel qu'il existe aujourd'hui est le résultat de différents bouleversements politiques survenus au $\mathrm{XX}^{\mathrm{e}}$ siècle. Trois grandes périodes jalonnent ces changements : l'avant Seconde Guerre mondiale, où la Pologne était sous régime capitaliste, l'après-guerre jusqu'en 1989 sous régime socialiste, et enfin l'après 1989 où l'économie de marché a été réinstaurée. Ces trois étapes n'ont pas seulement marqué les formes de l'habitat, mais ont notamment remanié la structure du parc de logements, de même qu'elles ont redéfini la notion de propriété des immeubles et des logements.

7 Avant la Seconde Guerre mondiale, le secteur privé dominait totalement le secteur de l'habitat, laissant peu de place aux autres formes juridiques d'habitation. En 1938, celuici représentait $95 \%$ des logements à Varsovie ; le reste du parc était propriété de l'État, 
des municipalités ou des coopératives d'habitation. Ces dernières étaient donc relativement rares représentant seulement $0,2 \%$ du parc de logements polonais (Cesarski, 2010). Selon leur mode de fonctionnement, les coopératives se décomposaient entre celles destinées aux employés modestes, et celles visant à construire des immeubles en propriétés collectives.

8 La Seconde Guerre mondiale a été un marqueur important dans l'histoire des villes en Pologne car un grand nombre d'entre-elles a été détruit. La capitale a notamment été la plus touchée avec $65 \%$ de ses bâtiments anéantis. En 1945, pour faciliter sa reconstruction, le gouvernement n'a pas hésité à communaliser une grande partie des biens immobiliers sur un peu plus de 14000 ha de son territoire. La même année, des pouvoirs de cantonnement ont été créés afin de gérer l'attribution de logements communaux dans les plus grandes villes polonaises. Bien que la plupart des biens privés soit devenue communale et le droit de disposition des logements et des immeubles très limité, la propriété privée n'avait pas disparu pour autant, même si son poids avait fortement décru.

9 À partir des années 1950, avec l'introduction du projet d'industrialisation du pays, l'importance du secteur de l'habitat et les dépenses liées à celui-ci ont fortement diminué (Andrzejewski, 1977). Afin de parer à la pénurie de logements qui commençait à s'instaurer, les coopératives d'habitation ont pris le relais de la construction à partir de la moitié des années 1960 sous l'égide de l'État, devenant ainsi les principaux promoteurs immobiliers dans les espaces urbains jusqu'au début des années 1990. La promotion des logements et leur gestion étaient le fait essentiellement des coopératives, des entreprises et des communes, restant ainsi sous étroit contrôle de l'État. Plus spécifiquement, les logements d'entreprise étaient construits par les entreprises d'État et les coopératives.

Durant la période socialiste, la Pologne faisait office d'exception au sein des pays d'Europe de l'Est, puisque son taux de propriétés privées était élevé au niveau national, notamment à cause de la décollectivisation de l'agriculture en 1956 (tableau 1). Cependant, le secteur privé de l'habitat en zone urbaine était devenu quant à lui faible, sa part diminuant jusqu'en 1989, même si en valeur absolue il a très faiblement augmenté durant cette période grâce à une ordonnance rendant possible l'acquisition des logements occupés à partir de 1972. Par ailleurs, ce fut seulement par la loi sur les coopératives de $1982^{1}$ que deux statuts d'occupation distincts ont été introduits : la location coopérative et la propriété coopérative. Le second permettait la transmission du bien immobilier ainsi que sa revente, excluant toutefois la copropriété des parties communes de l'immeuble qui restent entièrement à la charge de la coopérative. Cette situation hybride a été qualifiée par WĘcławowicz et Gaudray-Coudroy (1998) de semipropriété. La construction de logements privés individuels s'est également accélérée vers la fin du socialisme, de sorte que leur part est passée de $26 \%$ en 1980 à $37 \%$ en 1990 (Herbst, WĘcławowicz, 1993). Ainsi, un peu plus de $15 \%$ du parc de logements dans l'ensemble des villes était considéré comme privé en 1990 (Ibid.). À l'inverse, la part des coopératives dans la construction a eu tendance à baisser tout au long des années 1980 . Cependant, à l'aube des changements économiques des années 1990, l'importance des coopératives d'habitation, des logements municipaux et d'entreprise était encore extrêmement prégnante (représentant $56,2 \%$ du parc du logement en 1990). Le poids de la structure socialiste du parc de logements a rendu difficile sa transition vers un secteur de l'habitat où la propriété privée est devenue centrale. 
Tableau 1 - Part des logements polonais selon le type de propriété de l'immeuble entre 1960 et 1988

\begin{tabular}{|l|l|l|l|l|l|l|}
\hline \multirow{2}{*}{ Année } & \multirow{2}{*}{ Total } & \multicolumn{5}{|l|}{ Part des logements (en \%) } \\
\cline { 4 - 7 } & & Privés & total & Etat & coopératives & autres \\
\hline 1960 & 100 & 66,9 & 33,1 & 21,6 & 5,2 & 6,3 \\
\hline 1970 & 100 & 58,0 & 41,4 & 22,6 & 6,9 & 11,9 \\
\hline 1978 & 100 & 49,6 & 50,2 & 21,6 & 16,9 & 11,7 \\
\hline 1988 & 100 & 43,5 & 56,3 & 19,4 & 24,3 & 12,6 \\
\hline
\end{tabular}

\section{Le renouveau de la propriété privée et du marché immobilier après 1989}

11 À partir de 1989, de nombreuses mesures ont été prises par l'État polonais afin d'effectuer la transition entre un régime socialiste et un régime capitaliste. Le rétablissement des droits de propriété et la remise en place d'un marché immobilier ont été deux étapes fondamentales dans le processus de restructuration de l'espace urbain polonais.

\section{Évolution du secteur de l'habitat de 1989 à 2011 dans l'ensemble des villes polonaises}

Le secteur de l'habitat n'a pas été réformé tout de suite. Dans un premier temps, ce sont les biens non résidentiels du secteur public, ainsi que ceux de l'Église et des organisations caritatives qui ont été privatisés. Le nouveau gouvernement a préféré développer une politique du logement exclusivement tournée vers les coopératives (Herbst, WĘcławowicz, 1993), si bien qu'entre 1990 et 1991, la part de ces logements a augmenté et que les processus de privatisation et d'accession à la propriété ont été retardés (Marcuse, 1996 ; Hegedüs, Tosics, 1998 ; Glock, 2007). Cette dernière s'accélère surtout à partir du milieu des années 1990 car, d'une part, les investissements immobiliers reprennent après la crise économique du début des années 1990, et d'autre part la politique de revente aux locataires s'amorce. Durant les années 2000, l'accession à la propriété dans l'ensemble des villes polonaises s'accélère grandement. De 2002 à 2011, la part des logements en propriété privée individuelle augmente de plus de $40 \%$ (illustration 1). À l'inverse, la part des logements coopératifs diminue de $38 \%$ et cette baisse concerne presque exclusivement les logements qui étaient en location coopérative. Enfin, la part des logements communaux a également subi une diminution conséquente particulièrement au milieu des années 1990 et n'a pas cessé de décroître jusqu'en 2011 pour ne pas dépasser $12 \%$ du parc immobilier. 
Illustration 1 - Composition du secteur de l'habitat dans l'ensemble des villes polonaises en 2002 et 2011

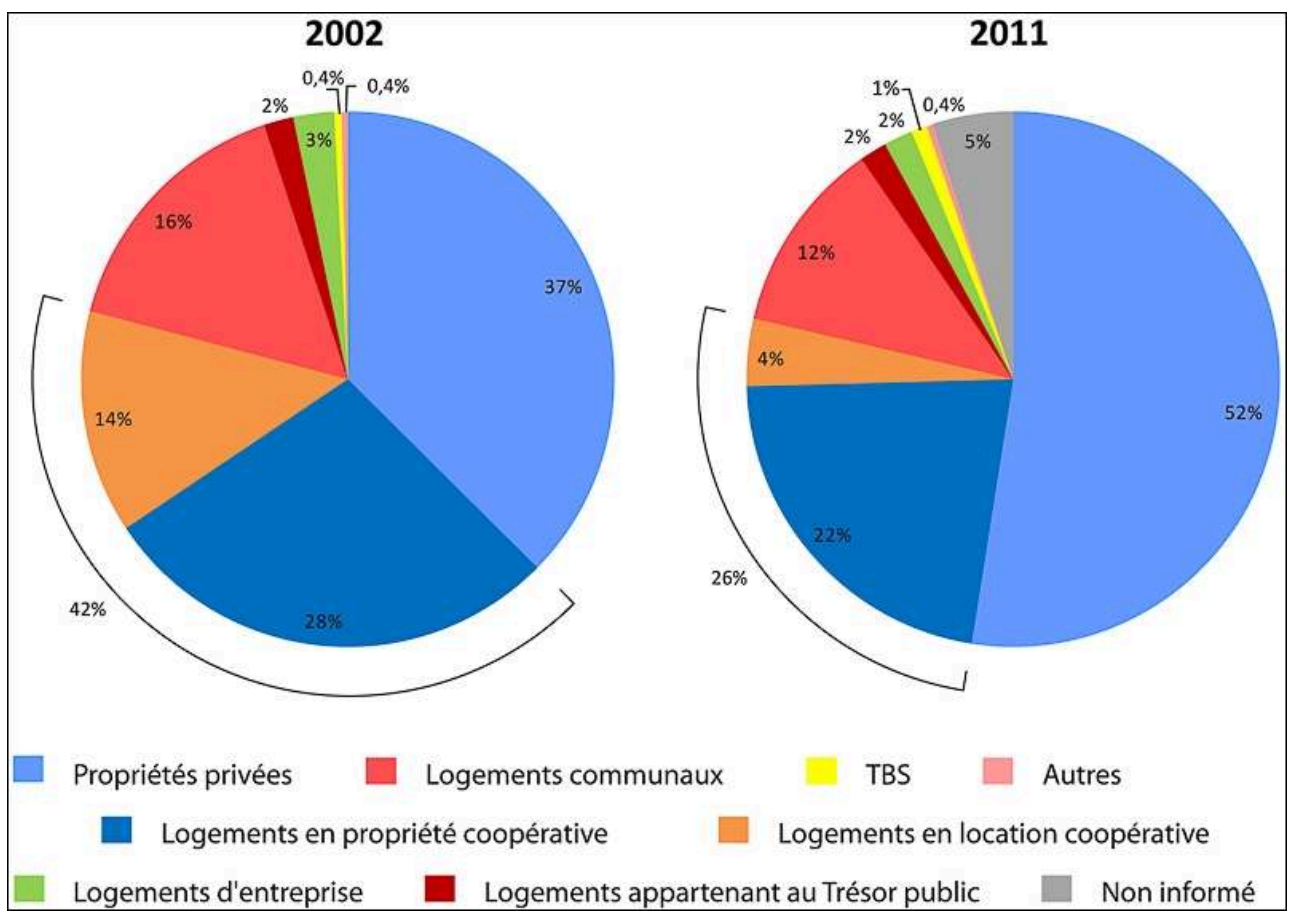

Source: GUS, Recensements de Population 2002 et 2011.

13 À Varsovie, où la communalisation sous le socialisme a été radicale, la structure du logement est spécifique, et la part du secteur privé a augmenté lentement dans les années 1990. Si la propriété privée individuelle représentait moins de $8 \%$ en 1988 (WĘcławowicz, Gaudray-Coudroy, 1998), elle restait encore peu significative au début des années 1990, alors que son poids n'a cessé de croître durant la seconde moitié de la décennie pour atteindre $34 \%$ en 2002. Ainsi, le poids des coopératives et des logements communaux était encore important en 1994, pour baisser de manière conséquente dès 1995 (illustration 2). 
Illustration 2 - Évolution de la structure du parc de logements à Varsovie entre 1994 et 2001

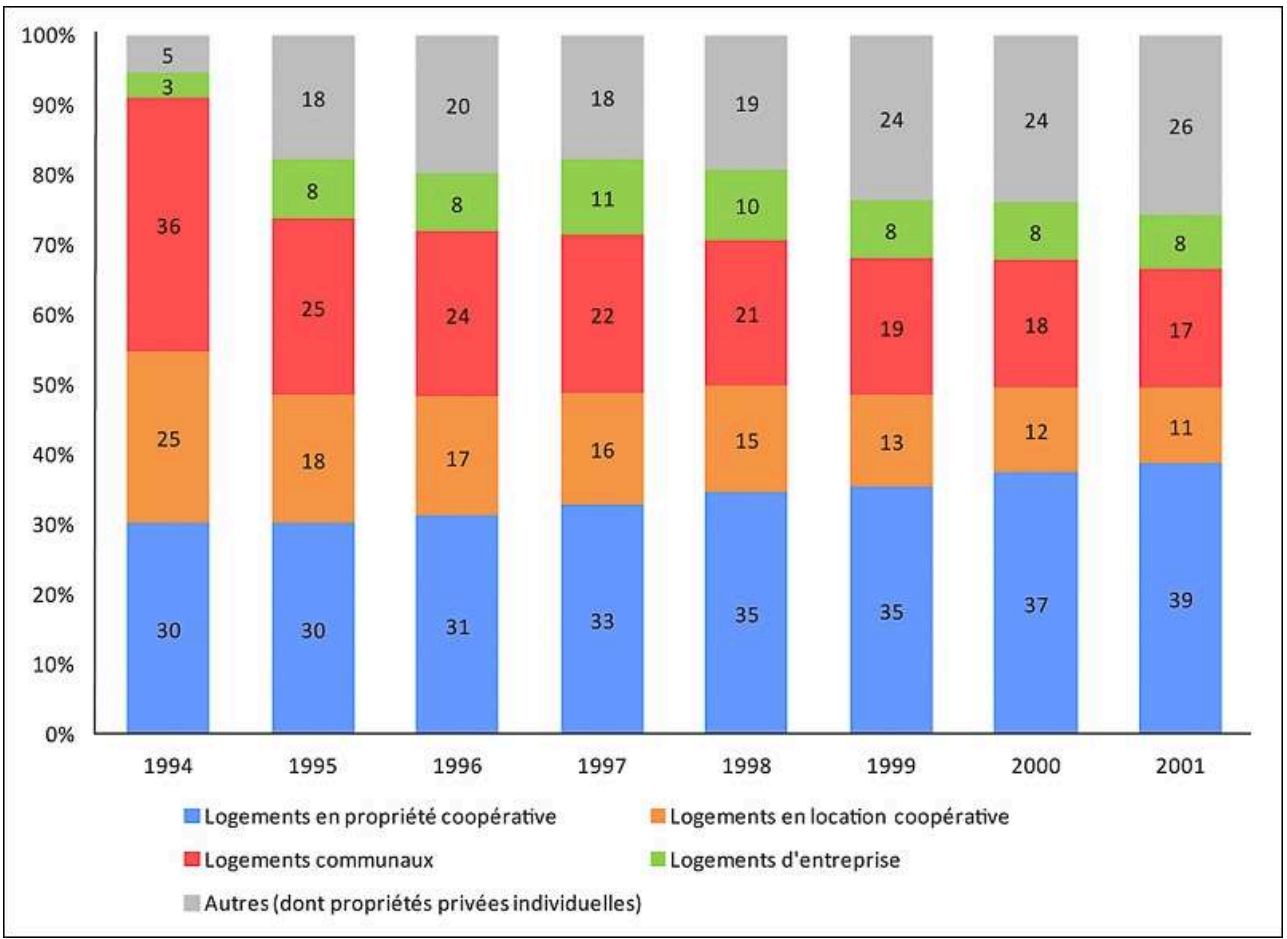

Source : GUS

Durant les années 2000, l'accession à la propriété progresse toujours sensiblement à Varsovie, mais contrairement à l'ensemble des villes polonaises, la part des logements en propriété privée et celle des coopératives en propriété fluctuent toutes deux entre $32 \%$ et $36 \%$. C'est le pourcentage de logements en location coopérative qui décroît le plus fortement, atteignant $9 \%$ en 2011, mais il reste élevé par rapport aux autres villes polonaises. Durant la même période, le stock des autres segments (d'État, communal, d'entreprise et des coopératives en location) reste quasiment inchangé même si leur part baisse très légèrement chaque année.

\section{Re-création d'un marché de l'immobilier permettant l'accession à la propriété}

Le marché immobilier en Pologne a été difficile à mettre en place. À la suite de près de trente ans de pénurie de construction de logements, le début des années 1990 n'a nullement constitué une reprise de la construction immobilière en Pologne (Coudroy de Lille, 2009), notamment parce que les financements des logements étaient rendus complexes par le passage d'une économie à l'autre (Gaudray, 1995), si bien que la construction immobilière a continué à chuter entre 1993 et 1996 (illustration 3). Le mètre carré en 1993 valait deux fois le salaire moyen d'un Polonais, et en 1992 de nombreux logements neufs sont restés vacants dont 12500 à Varsovie (Ibid.). 
Illustration 3 - Nombre annuel de logements construits de 1991 à 2013 en Pologne

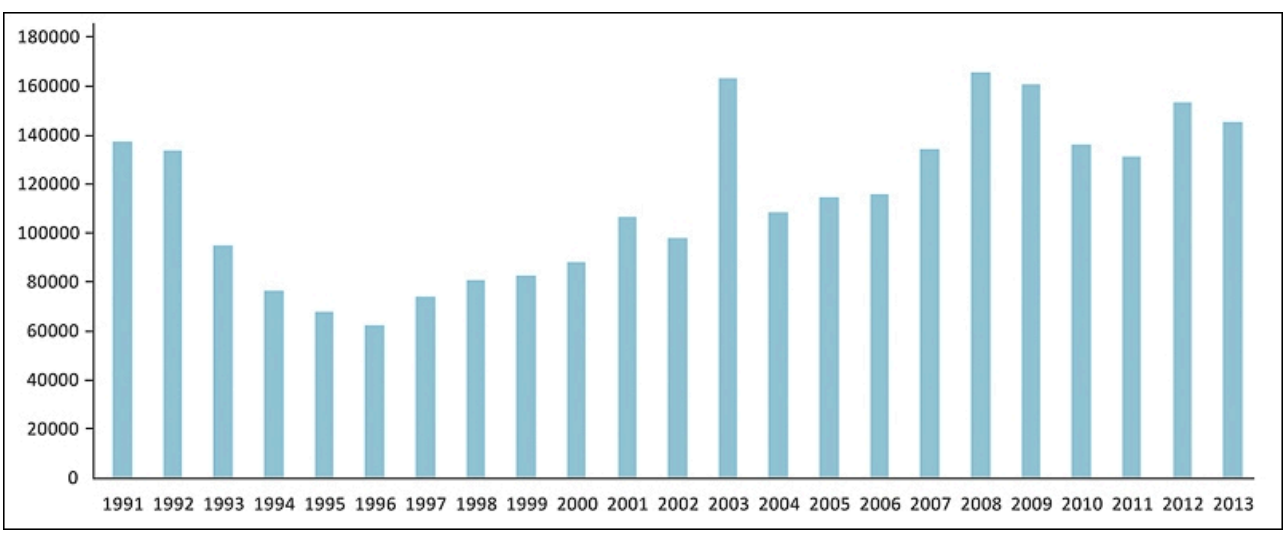

Source : GUS, 2014

Ainsi, au début des années 1990, le marché de l'immobilier fonctionnait principalement pour les secteurs productifs grâce à la privatisation des locaux d'entreprise d'État et la construction d'immeubles de bureaux, de locaux commerciaux et industriels. Vers la fin des années 1990, alors que la restructuration du secteur de l'habitat s'est engagée, la crise de la construction de logement s'est quelque peu atténuée (Coudroy de Lille, 2009). Le marché a alors intégré la construction de nouveaux logements, puis quelque temps après a englobé la privatisation des logements coopératifs et communaux. Néanmoins, la part des propriétaires polonais restera moindre en comparaison des autres pays anciennement socialistes en Europe, notamment par le maintien du statut de locataire au sein du parc coopératif et la mise en place d'un habitat social des Sociétés de Construction Sociale ${ }^{2}$ (TBS) dès 1995 (Coudroy de Lille, 2012). Cette création de marché a permis la pénétration de capitaux étrangers dans le pays et a ainsi dynamisé l'économie (Zborowski, 2005). Il faudra attendre la fin des années 1990 et surtout le début des années 2000 pour que le marché immobilier résidentiel s'améliore grâce notamment à l'arrivée de promoteurs immobiliers étrangers. À titre d'exemple, $74 \%$ des logements livrés entre 2002 et 2012 à Varsovie sont construits par des promoteurs immobiliers (BDL, GUS). Malgré tout, la pénurie de logements sur le marché est toujours importante. L. Coudroy de Lille (2012) souligne la faiblesse du taux de construction au niveau national jusqu'à la fin des années 2000, celui-ci ne dépassant jamais les 5 pour 1000 que ce soit à l'échelle nationale ou à celle des villes. Cela s'explique en partie par la faiblesse des investissements vers le secteur de l'habitat en constante baisse, passant de 4,8\% dans le budget de l'État en 1990 (Cesarski, 2007) à $0,5 \%$ en 2012 (Zaniewska et al., 2013).

17 Toutefois, durant vingt ans, la part des actes notariés concernant la vente d'un logement par un individu privé n'a cessé d'augmenter, passant de $23 \%$ en 1990 à $51 \%$ en 2009 , et même $61 \%$ des actes en $2006^{3}$. Ces transactions sont d'autant plus impressionnantes qu'entre 1990 et 2009 , leur nombre a été multiplié par quatre ${ }^{4}$. En outre, les actes notariés concernant la vente de logements par l'État ont pris de l'importance à partir de 1993 jusqu'en 2004, représentant environ $15 \%$ de ces actes sur toute la période.

Bien que la production de logements et le marché de l'immobilier en Pologne ne cessent de se développer, l'accession à la propriété devient coûteuse pour les ménages, notamment dans les grandes villes. Afin d'élargir les possibilités d'accession au 
logement, l'État a adopté le programme «La famille chez soi » entre 2006 et 2013. Durant huit ans, les ménages accédant à la propriété dans le cade de ce programme bénéficiaient du financement d'une partie des intérêts dont le crédit avait été souscrit en zloty ${ }^{5}$ auprès d'une banque partenaire de la Banque Nationale (Bank Gospodarstwa Krajowego). Tous les ménages pouvaient participer au programme sans distinction de revenus à condition qu'ils ne soient pas déjà propriétaires d'un bien immobilier. Seuls la taille du logement et le prix du mètre carré étaient limités et définis par l'État. Les ménages de la classe moyenne supérieure ont également très largement profité de cette politique. À partir de la crise de 2008 , ce programme a été particulièrement opportun pour les promoteurs immobiliers privés, leur permettant de vendre les logements construits malgré un marché immobilier en difficulté. Le nombre de crédits attribués aux ménages est ainsi passé de 4000 en 2007 à plus de 45000 en 2012 (Bank Gospodarstwa Krajowego). Depuis 2014, ce programme a été remplacé par «Un logement pour les jeunes» se focalisant sur l'accession à la propriété des moins de 35 ans.

19 Néanmoins, au-delà de ces mécanismes classiques d'accession à la propriété, le poids de la structure socialiste a considérablement complexifié le statut juridique de la propriété privée rendant sa lecture difficile, et ce tâtonnement législatif a grandement retardé l'accession à la propriété.

\section{Les différents modes d'accession à la propriété dans le parc construit avant 1989}

Plusieurs changements législatifs ont permis l'accession à la propriété privée d'une partie de la population. Il a cependant fallu s'adapter à chaque segment de l'habitat public qui n'impliquait pas les mêmes réformes.

\section{La privatisation des logements communaux et d'entreprise : une politique globale d'accession à la propriété}

21 La structure d'occupation au sein du parc de logements communaux a fortement changé en raison de sa privatisation. Les collectivités locales (communes), créées en 1990 et auxquelles incombe désormais la responsabilité de ces logements (entretien, modernisation et construction), ont vendu une grande part du stock de logements. On estime qu'au début de la transformation du système, 650000 logements communaux (un tiers du parc communal) ont été vendus pour $10 \%$ du prix du marché, permettant aux ménages aux revenus modestes d'accéder à la propriété (Zaniewska, 2005). À cette catégorie, il faut ajouter les logements qui dépendaient des entreprises d'État. La liquidation de ces dernières dans les années 1990 a fait chuter fortement le nombre de logements d'entreprise, passant d'environ 1,4 million en 1993 à 256000 en 2002 (soit une diminution de $82 \%$ ). Ces logements ont été en grande partie privatisés ou ont été intégrés à d'autres entités (aux communes ou au Trésor Public) (Ministère des Transports et de la Construction, 2005).

Par ailleurs, dans le but de résorber les besoins de logements pour les classes moyennes qui ne remplissent pas les conditions d'attribution d'un logement communal et ne disposent pas d'un revenu suffisant pour acheter un logement sur le marché, les TBS 
ont été créées en 1995. Cependant, ces logements «sociaux » ne sont pas si abordables qu'il y parait et n'ont pas eu le succès escompté, car les demandeurs doivent payer à l'entrée une contribution qui atteint parfois $30 \%$ du prix du logement. Par ailleurs, l'accession à la propriété a également été rendue possible à l'intérieur de ce segment, complexifiant encore un peu plus le statut juridique de la propriété privée en Pologne.

\section{L'hybridité du système coopératif : une accession à la propriété complexe}

Le rôle et les modes d'attribution des logements par les coopératives d'habitation ont beaucoup évolué depuis leur création et se sont compliqués ces dernières années. Durant le socialisme, les logements coopératifs revêtaient deux formes distinctes : en location coopérative ou en propriété coopérative dit aussi semi-propriété. La situation évolue en 1994 lors de la promulgation d'une loi qui introduit entre autres la propriété distincte d'un local (pl. odrębna własność lokalu) et permet de créer des syndicats de copropriété afin d'assurer la gestion d'immeubles entre propriétaires, lesquels peuvent être des gestionnaires institutionnellement différents. En conséquence, la structure d'occupation des logements dans les immeubles gérés par les coopératives d'habitation s'est diversifiée et actuellement, elle se déploie en quatre formes distinctes ${ }^{6}$ :

- Les logements en location coopérative : sous cette forme une contribution financière est exigée à l'entrée, le locataire est membre de la coopérative et les logements ne sont ni transmissibles ni aliénables;

- Les logements en propriété coopérative : ceux-ci peuvent être transmis et aliénables ;

- Les logements en location : il s'agit d'une location simple passant par la coopérative; les locataires ne sont pas membres de la coopérative, ne paient pas de contribution à l'entrée mais seulement une caution ;

- Les logements en propriété individuelle : les individus sont propriétaires du logement et des parties communes de l'immeuble; ils deviennent ainsi copropriétaires de l'immeuble au sein du syndicat de copropriété.

Cette diversité des statuts d'occupation dans les coopératives provient en partie du statut de propriété du foncier. Si une coopérative d'habitation ne dispose pas des droits de propriété ou de bail emphytéotique des parcelles sur lesquelles les immeubles coopératifs sont installés, la propriété individuelle des logements ne peut pas être établie. Ainsi, beaucoup d'habitants souhaiteraient posséder leur logement en propriété individuelle, mais sont obligés d'attendre le changement d'occupation foncière. Entre 1988 et 2002, la part des logements en location coopérative a diminué de plus de $47 \%$, passant d'environ 2,3 million à 1,1 million (Ministère des Transports et de la Construction, 2005). Cette baisse s'explique par la faible part de logements neufs construits en coopératives, mais aussi par une croissance des accédants à la propriété dans ce parc. Par exemple à Varsovie, entre 1991 et 2010, plus des deux tiers des logements coopératifs sont devenus des logements en propriété individuelle (Górczyńska, 2012a). Ainsi, les promoteurs immobiliers de la période socialiste sont devenus les gestionnaires du parc en location durant la période post-socialiste. 


\section{La restitution des biens immobiliers d'avant-guerre}

La restitution des biens communalisés d'après-guerre à leurs propriétaires d'origine pose de nombreux problèmes et a été maintes fois débattue au sein du gouvernement mais aucune loi n'a été votée à cet effet. La capitale et les villes détruites en grande partie durant la Seconde Guerre mondiale sont peu concernées par ce phénomène, mais c'est un enjeu fondamental pour les villes telles que tódź ou Cracovie (Glock, 2007). Certains auteurs avancent le chiffre de 33000 restitutions entre 1990 et 1994, ce qui représenterait $1 \%$ du stock de logements communaux durant cette période (Hegedüs, Tosics, 1998). Plus récemment, ces restitutions ont pris de l'ampleur dans certaines villes. Ainsi les restitutions concerneraient environ $20 \%$ des immeubles anciens du centre-ville de Cracovie (Glock, 2007). À Varsovie, le nombre de biens revendiqués augmente depuis les années 1990 (illustration 4). Plus de 2000 demandes sont actuellement en cours de procédure et nécessiteront parfois plusieurs années pour que la restitution soit effective.

Illustration 4 - Nombre de biens communalisés à Varsovie dont les anciens propriétaires ont acquis le droit d'usufruit perpétuel entre 1990 et 2012

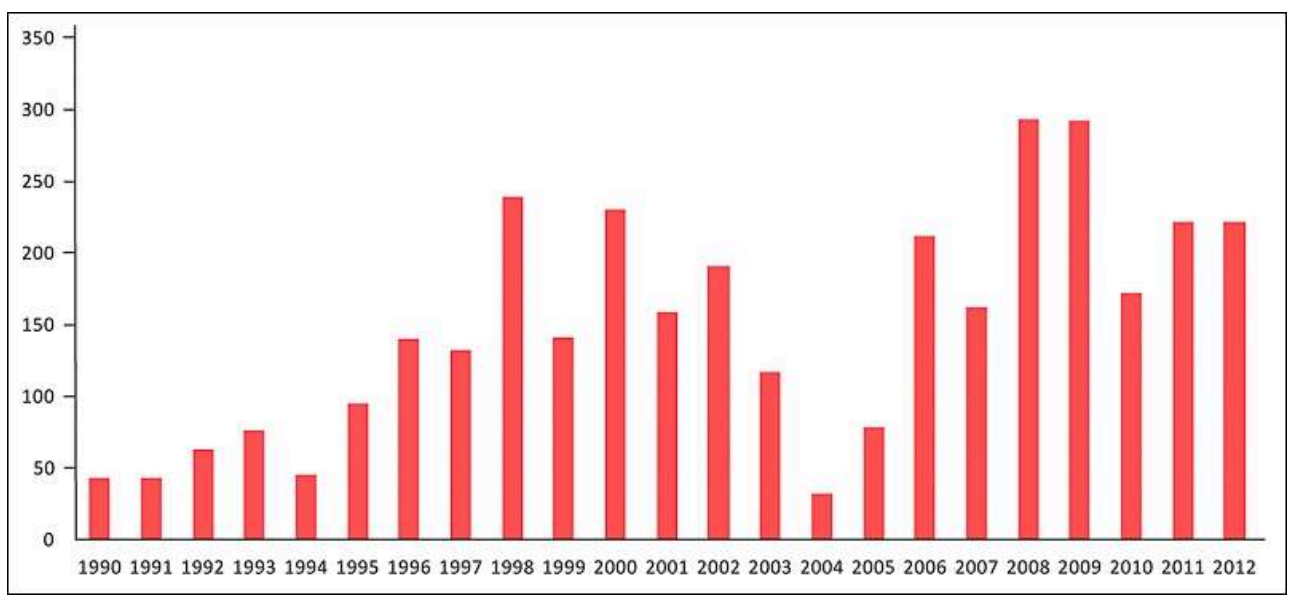

Source: Mairie de Varsovie, 2014.

Une petite partie du parc de logements anciens a été privatisée des années 1970 jusqu'en 1989 (Górczyńska, 2014b). À partir des années 1990, les demandes de restitutions ont commencé à concerner des immeubles entiers, si bien qu'après restitution au propriétaire d'origine (ou à ses héritiers), les locataires communaux deviennent de fait des locataires privés. Toutefois, la restitution des biens ne destitue aucunement les locataires qui avaient préalablement acheté leur logement. La superposition des différents statuts d'occupation des logements (propriétaires et anciens locataires communaux) a conduit à une situation hybride de gestion de ces immeubles (Górczyńska, 2013).

27 La restitution de biens immobiliers devient également lucrative pour les promoteurs immobiliers, car les individus qui essayent de récupérer ces biens sont souvent âgés, habitent parfois à l'étranger ou n'ont pas les connaissances juridiques pour suivre la procédure de restitution et préfèrent alors les revendre. C'est ainsi que les promoteurs rachètent soit les immeubles restitués, soit les demandes de restitution. Les immeubles réhabilités par les promoteurs constituent une part très faible du marché de 
l'immobilier encore à l'heure actuelle. Ils sont néanmoins très recherchés par les classes aisées (Bouloc, 2013). Ainsi, cette partie du parc de logement constitue un vivier de résidences de luxe potentiel dont la situation centrale ne fait que renforcer l'attraction.

\section{Les conséquences de l'accession à la propriété dans l'espace urbain : exemple de Varsovie}

La diversification des modes d'accession à la propriété provoque un certain nombre de conflits entre groupes sociaux à l'échelle des immeubles, mais aussi à celle des quartiers. Les conséquences sont également d'ordre spatial, notamment parce que les différents segments du secteur de l'habitat se répartissent inégalement dans l'espace. À travers l'exemple de Varsovie, il est possible d'éclaircir certaines de ces confrontations.

\section{Les logements pré-socialistes : un nouvel âge d'or?}

À Varsovie, la majorité des logements anciens se situent dans les quartiers centraux, mais leur qualité diffère suivant leur localisation sur la rive droite ou gauche de la Vistule. Le patrimoine immobilier dans le centre-ville (Śródmieście) situé sur la rive gauche est en meilleur état, de sorte que de nombreux logements y sont privatisés (WĘcławowicz, 1998) et représentent $16 \%$ de tous les immeubles revendiqués à Varsovie $^{7}$ (illustrations $5 \mathrm{a}$ et $5 \mathrm{~b}$ ). À l'inverse, Praga Północ situé sur la rive droite présente un parc immobilier plus dégradé dont les logements communaux étaient attribués généralement à des individus aux revenus plus modestes. La privatisation existe dans ce quartier mais y est beaucoup plus lente, peu d'immeubles ont jusque-là été réhabilités et on y compte seulement $6 \%$ des immeubles revendiqués (illustration 6). 
Illustration 5a - Réhabilitation luxueuse d'un immeuble en centre-ville (Śródmieście) : façade restaurée

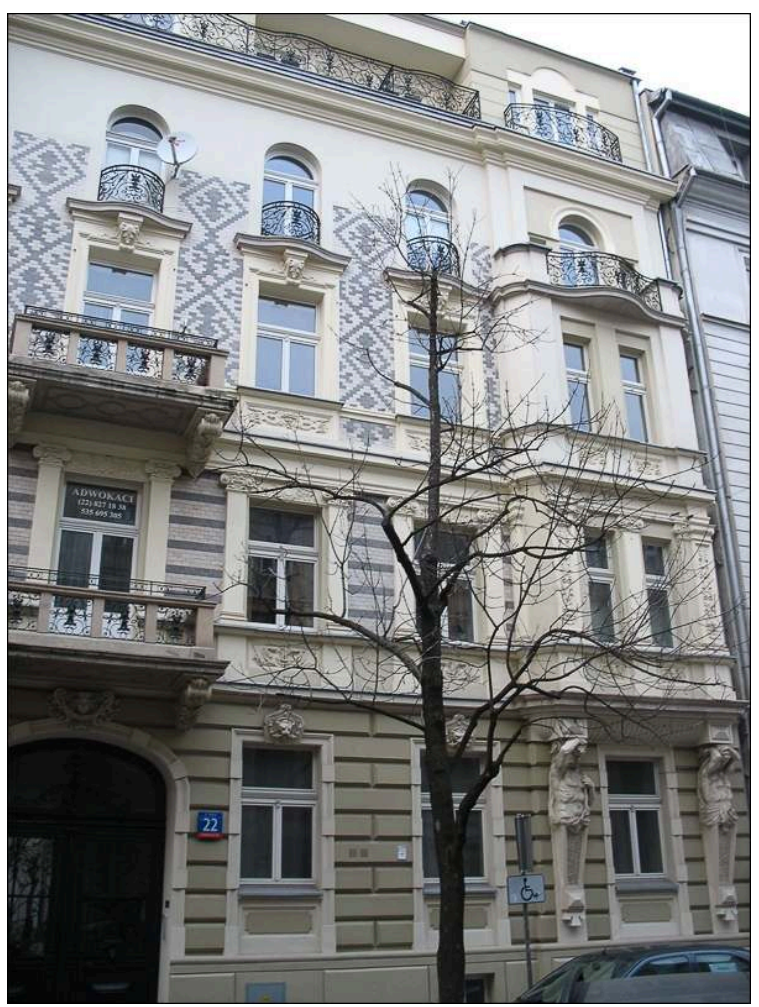

Auteur : M. Górczyńska, 2012. 
Illustration 5b - Réhabilitation luxueuse d'un immeuble en centre-ville (Śródmieście) : cour restaurée

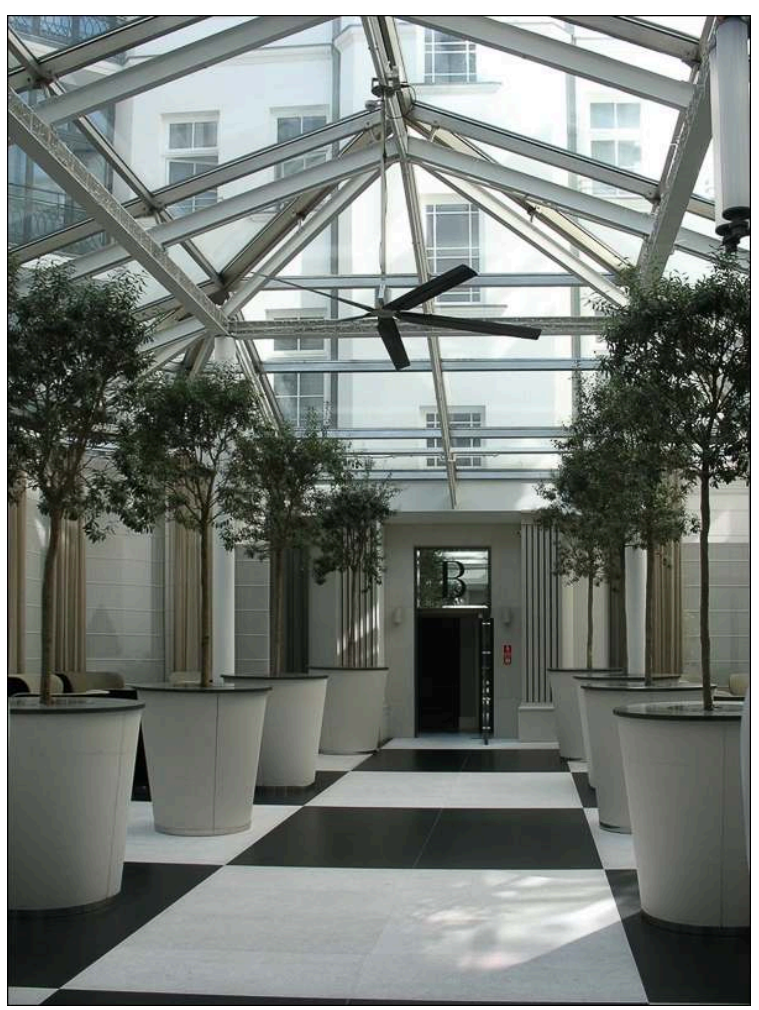

Auteur : M. Górczyńska, 2014 
Illustration 6 - Différence en termes de réhabilitation à Praga Północ (rue Ząbkowska)

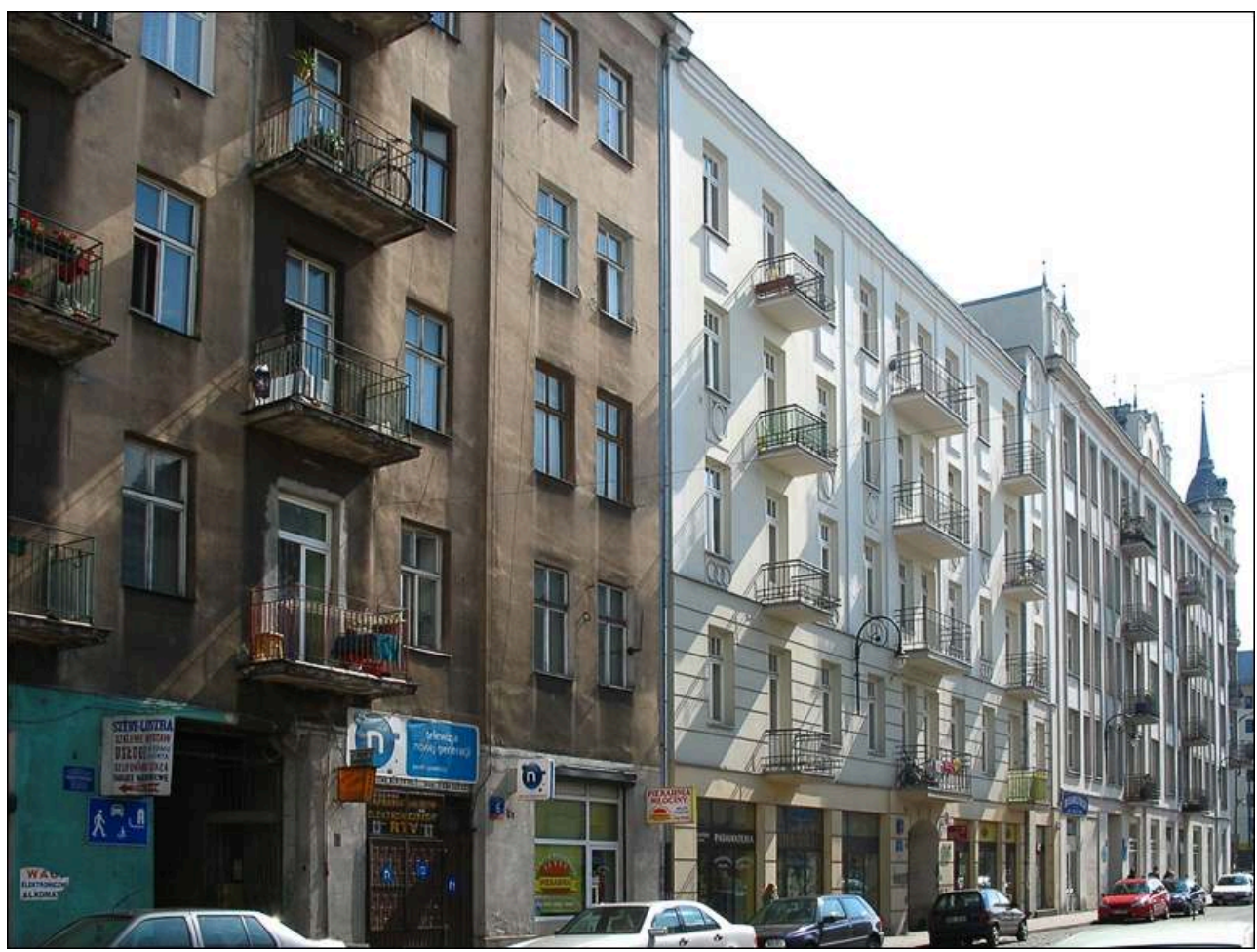

L'immeuble au premier plan est en cours de revendication tandis que l'immeuble au fond à droite a été réhabilité par la municipalité.

Auteur : M. Górczyńska, 2012

La multiplication des statuts de propriété à travers la privatisation de logements et la restitution d'immeubles (propriétaires institutionnels, propriétaires individuels et promoteurs immobiliers) est souvent source de conflits. L'acquisition d'un immeuble anciennement communal par un individu représente la situation la plus conflictuelle. En effet, cela implique un changement de statut des logements qui passent d'une location communale à une location privée, et modifie de ce fait le droit de location. Le nouveau propriétaire établit les loyers en fonction du prix du marché et ceux-ci peuvent donc devenir insoutenables pour les ménages qui y résident, souvent âgés (Górczyńska, 2014a). Les expulsions sont fréquentes et certains nouveaux propriétaires profitent du manque de connaissance de leurs droits par les locataires pour agir de manière peu scrupuleuse. Par exemple, les propriétaires ne couvrent plus les frais d'entretien ou de réparation nécessaires ou encore coupent les arrivées d'eau et de gaz, aggravant le mal-être des locataires et leurs conditions de vie. Face à cette situation, les autorités locales tentent de leur côté de reloger ces locataires dans de nouveaux logements communaux; mais la demande de logements dépassant l'offre, ces problèmes sont rarement résolus. Certains locataires concernés réagissent en créant des associations ou des regroupements de locataires comme par exemple le «Comité de défense des locataires ${ }^{8}$ ». Ces initiatives n'ont cependant qu'un rôle très restreint d'information de leurs droits auprès des locataires et les dénonciations faites par ces mouvements n'ont eu pour le moment qu'un faible impact médiatique. 


\section{Le parc de logements socialiste : des grands ensembles mi-publics mi-privés}

Le régime socialiste a produit différents types de logements tant d'un point de vue architectural qu'en terme de qualité des matériaux, de sorte que certains sont encore aujourd'hui de bonne qualité, ont parfois été rénovés et d'autres sont extrêmement détériorés. Ces différences proviennent des politiques de construction qui se sont succédées durant cette période. Dans les années 1950, le complexe résidentiel Marszałkowska Dzielnica Mieszkaniowa (MDM) dont l'architecture imposante devait représenter la force de l'idéologie socialiste selon la volonté politique de reconstruction de la capitale fut érigé (illustration 7). Les objectifs de la politique du logement à partir des années 1960 se sont fondés sur l'augmentation de la densité des espaces déjà bâtis afin d'aménager les terrains constructibles autant que possible mais souvent au détriment de la qualité des ensembles préfabriqués et des logements. Au tournant des années 1960 et 1970, suivant l'idéologie urbanistique socialiste, les nouvelles constructions résidentielles ont été placées en périphérie sous la forme de grands ensembles dont l'architecture était simple, préfabriquée et bien éloignée des premiers investissements réalisés en centre-ville après la guerre (illustration 8). La qualité et la structure d'occupation des logements construits durant le socialisme dépendent donc de leur période de construction, de sorte que certains grands ensembles restent attractifs encore aujourd'hui sur le marché de l'immobilier (Celińska-Janowicz, 2010) et d'autres ne le sont pas du tout. Bien évidemment, la localisation de ce parc et l'accès aux infrastructures sociales, de transport et culturelles influencent également considérablement leurs prix.

Illustration 7 - Place de la Constitution à Varsovie

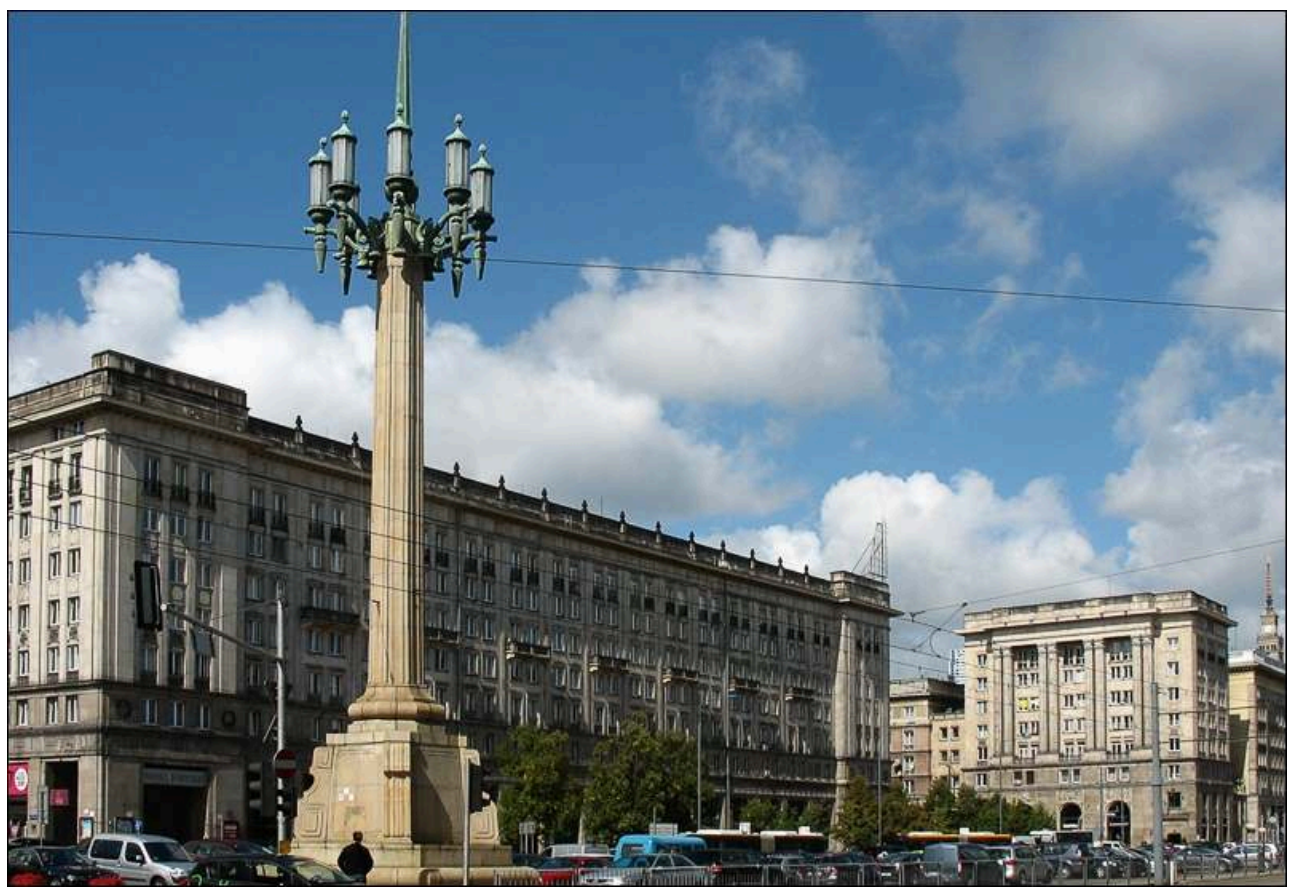

Auteur : M. Górczyńska, 2014. 


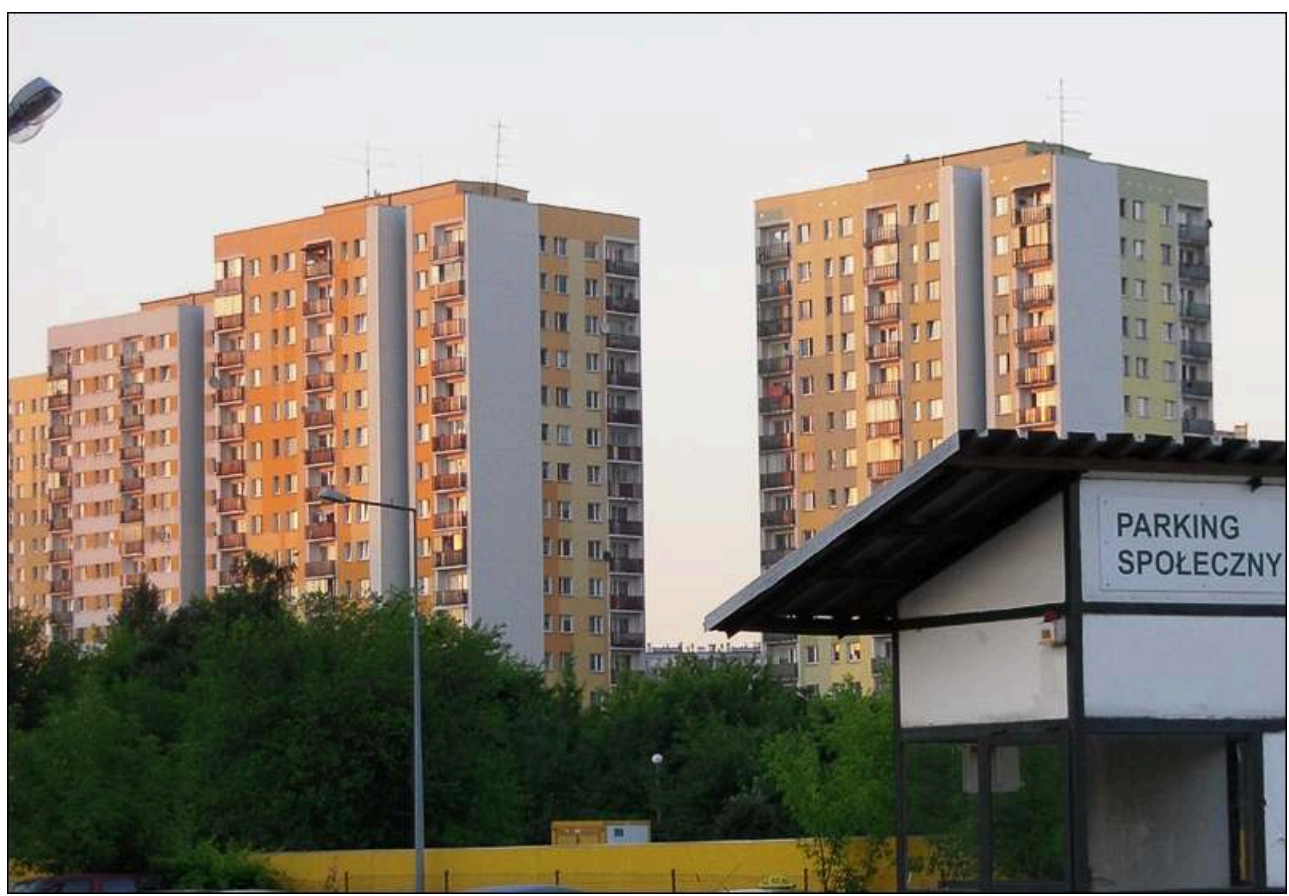

Auteur : M. Górczyńska, 2010

Depuis 1989, ce parc de logements soulève certains problèmes. D'une part, la privatisation qui englobe souvent une partie seulement des logements dans un immeuble (surtout dans les immeubles municipaux) a conduit à la création de copropriétés mixtes, semi publiques, semi-privées, parfois difficiles à gérer. D’autre part, l'accession à la propriété des logements à des prix très bas a conduit à la diversification socio-économique des membres de ces nouvelles copropriétés. Par ailleurs, les espaces communs n'appartiennent plus uniquement aux collectivités locales, mais également à des personnes physiques, de sorte que les prises de décisions peuvent être conflictuelles car chaque protagoniste ne défend pas les mêmes intérêts. Enfin, les possibilités financières de couvrir les frais de réhabilitation de l'immeuble sont pour certains propriétaires très réduites, permettant seulement l'entretien élémentaire, ce qui conduit à une détérioration du patrimoine en propriété privée (par exemple, certains propriétaires ne sont pas en mesure de participer financièrement à l'achat de nouvelles fenêtres). De ce fait, dans le paysage urbain, les situations sont très disparates d'un immeuble à l'autre et quelquefois dans une même résidence. Certains immeubles ont été rapidement rénovés, car la majorité des logements sont devenus privés (illustration 8), d'autres ne le sont toujours pas. 


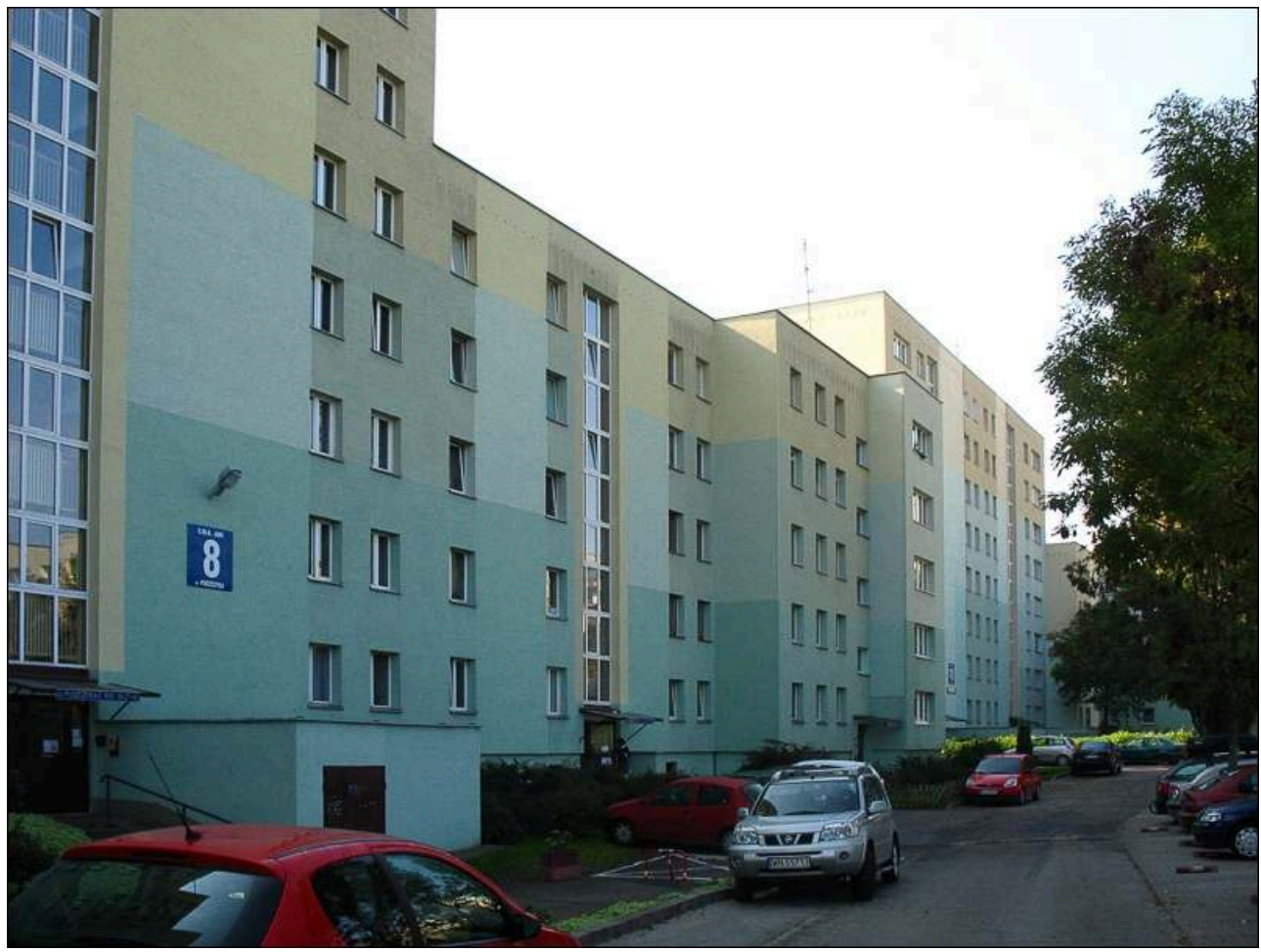

Auteur : M. Górczyńska, 2010

D'autre part, les logements restant en location qu'ils soient municipaux (ou coopératifs, soufrent d'arriérés de paiement de loyer, rendant les frais d'entretien encore plus difficiles à couvrir par les copropriétés. Cette situation touche particulièrement le stock coopératif où durée et nombre des arriérés sont plus élevés.

\section{Les logements construits après 1989 : une accession rapide à la propriété privée}

34 La construction de nouveaux logements dans l'ensemble des villes polonaises a permis aux ménages dont le statut économique progressait durant les années 1990 d'accéder rapidement à la propriété privée sans grande complication juridique ou statutaire. À Varsovie, ces constructions ont réellement pris de l'essor durant les années 2000, grâce à la diversification de l'offre de logements neufs des promoteurs immobiliers (Górczyńska, 2012b, Bouloc, 2013), allant de la tour d'une cinquantaine d'étages (illustration 10), en passant par la résidence fermée en habitat collectif (illustrations 11a et 11b) ou à la maison individuelle (illustration 12). 
Illustration 10 - La tour « Złota 44 » en centre-ville

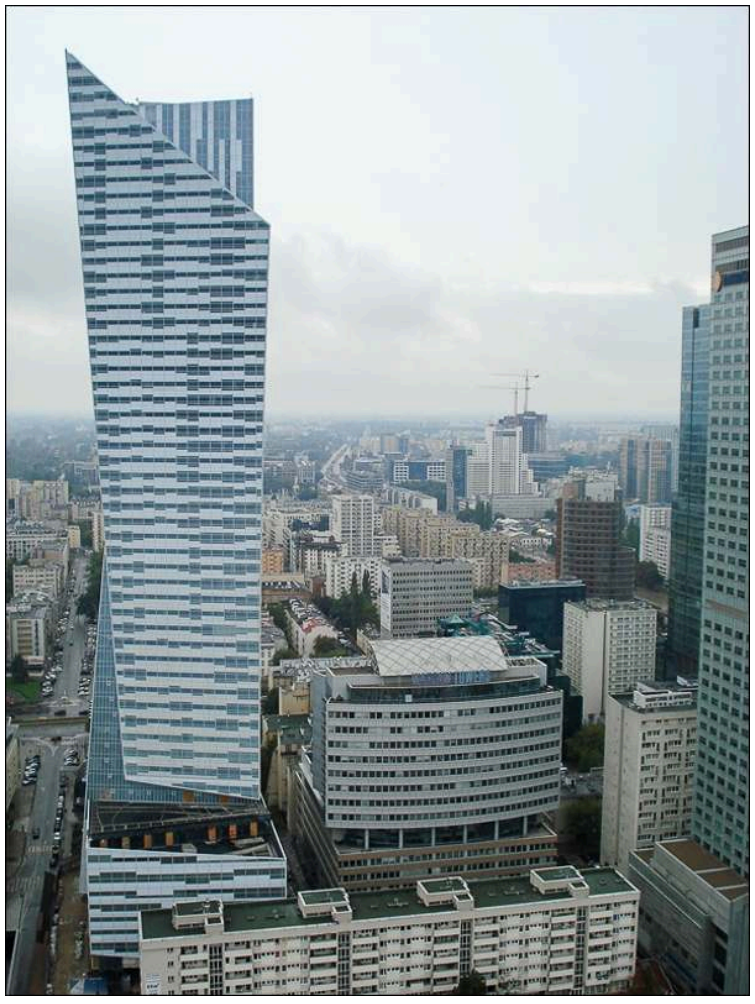

Auteur : C. Bouloc, 2014

Illustration 11a - Résidence fermée Marina dans le quartier de Mokotów : entrée

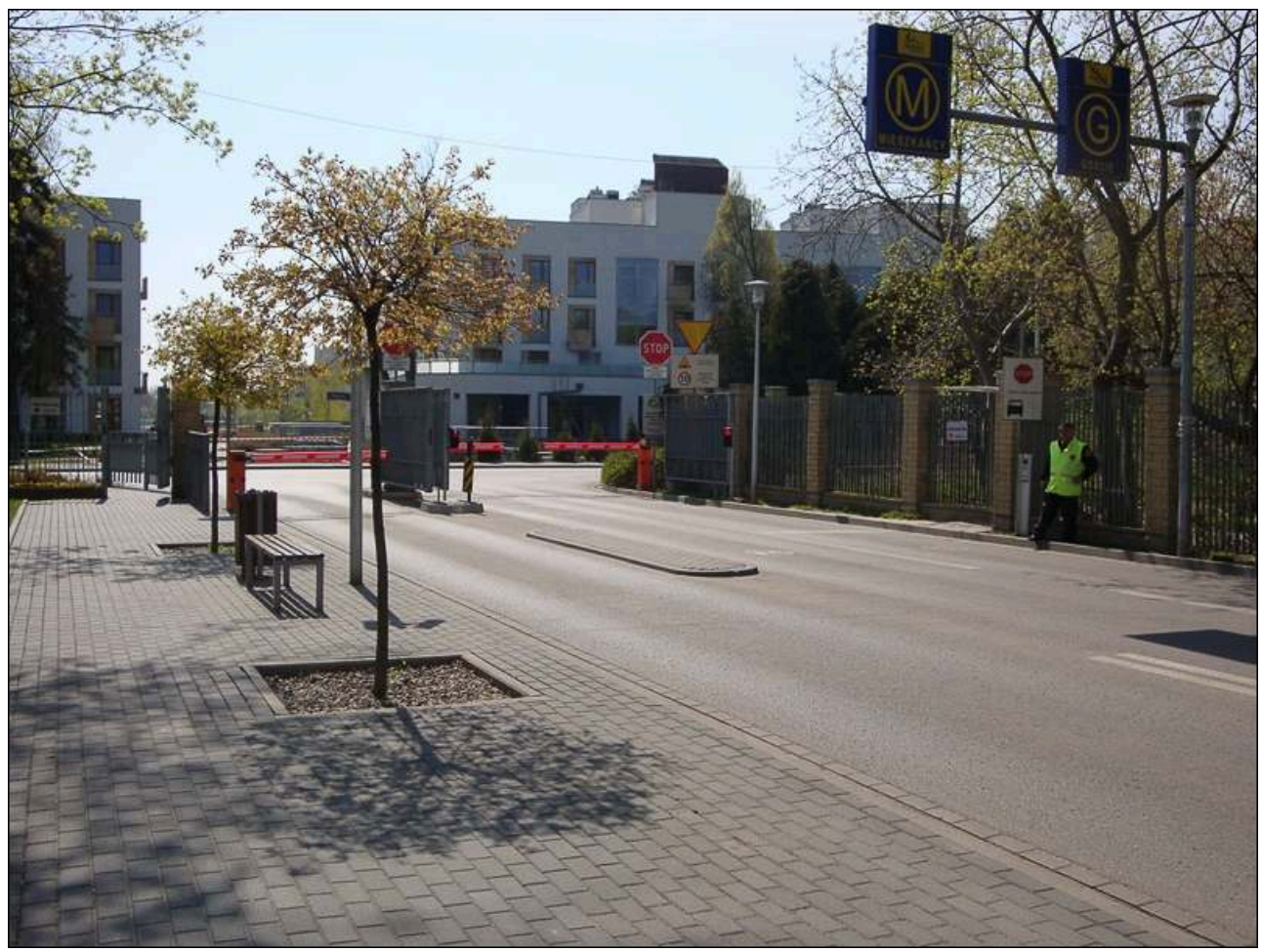

Auteur : C. Bouloc, 2011 
Illustration 11b - Résidence fermée Marina dans le quartier de Mokotów : clôtures à l'intérieur de la résidence délimitant un ensemble d'immeubles et de pavillons

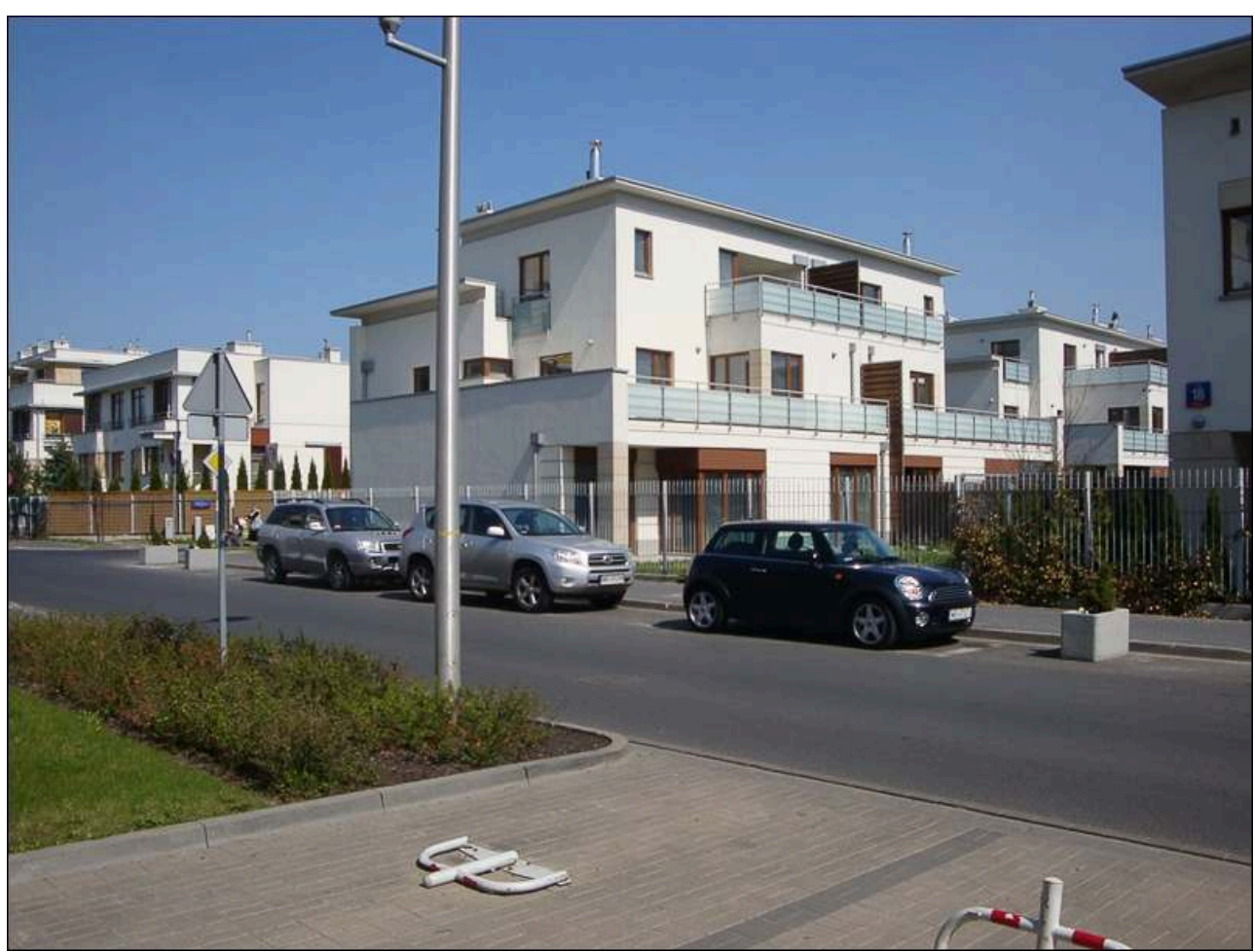

Auteur: C.Bouloc, 2011.

Ces nouvelles constructions résidentielles se sont majoritairement concentrées dans les quartiers périphériques de la ville, là où les promoteurs immobiliers pouvaient débloquer de larges parcelles pour construire des résidences, mais aussi où les ménages pouvaient acquérir une parcelle plus grande pour construire une maison individuelle, un bien quasi-inaccessible durant le socialisme. 
Illustration 12 - Exemple d'habitat neuf individuel dans le quartier de Wawer (Anin)

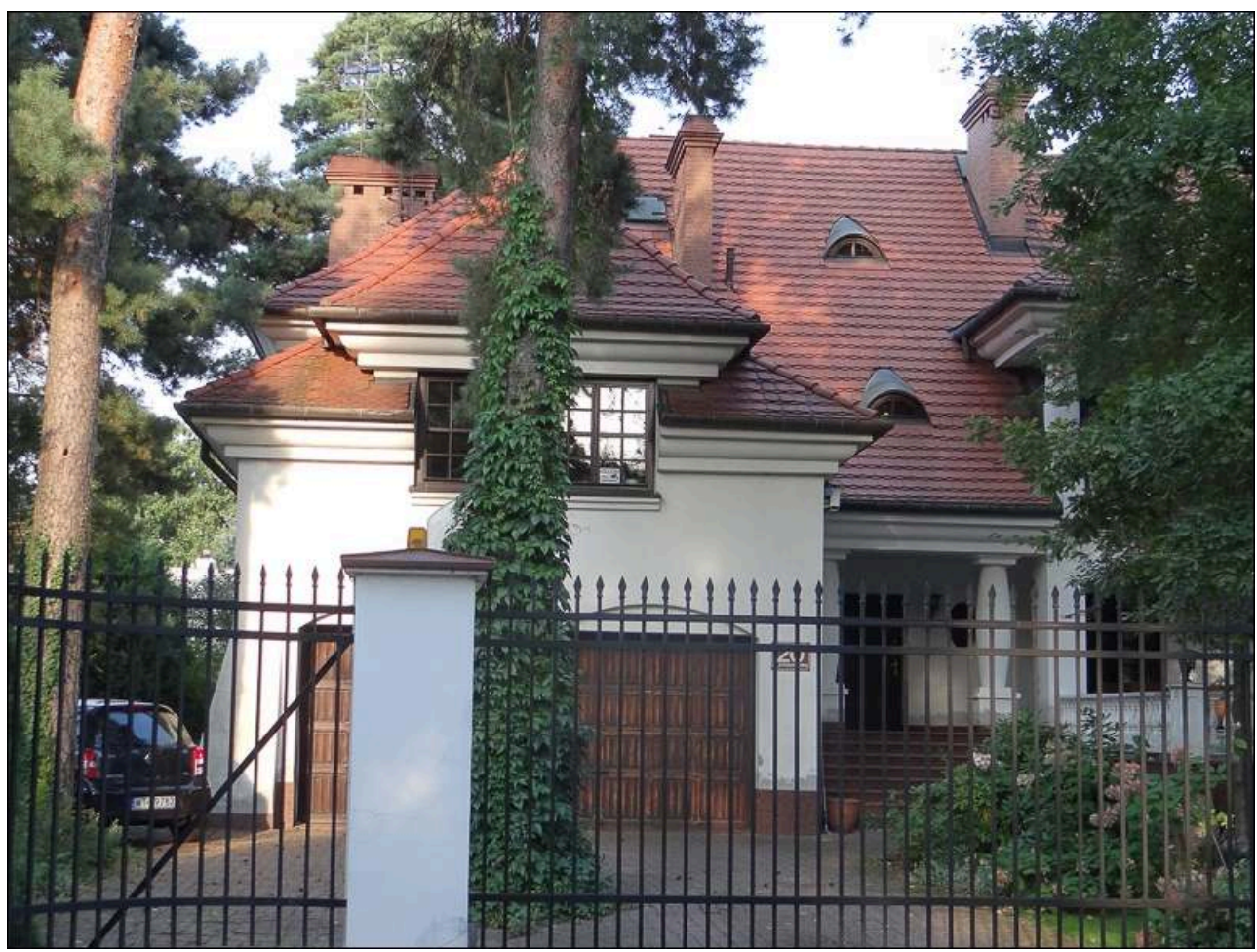

Auteur: C. Bouloc, 2014

À Varsovie, les quartiers périphériques ont connu un développement important au cours de ces dix dernières années. Par exemple, les quartiers BiałołŁ̨ka et Wilanów disposent d'un parc de logements construit pour plus de la moitié entre 2002 et 2012 (illustration 13), suivis de Wesoła, Bemowo et Ursus où plus du quart du parc résidentiel date de cette même période. Par ailleurs, les quartiers les plus excentrés au sud-est de la ville, encore forestiers et agricoles, accueillent majoritairement un nouvel habitat en maison individuelle. C'est le cas notamment de Wawer où plus de la moitié des nouvelles constructions sont des investissements individuels ou encore de Rembertów et Wesoła où ces investissements individuels représentent entre un quart et la moitié des logements neufs. En général, les quartiers périphériques (en couleurs foncées sur la carte) accueillent plus de $25 \%$ des logements neufs sur leur territoire. 
Illustration 13 - Carte présentant la part des logements neufs construits et des investissements réalisés par des personnes physiques par quartiers entre 2002 et 2012 à Varsovie

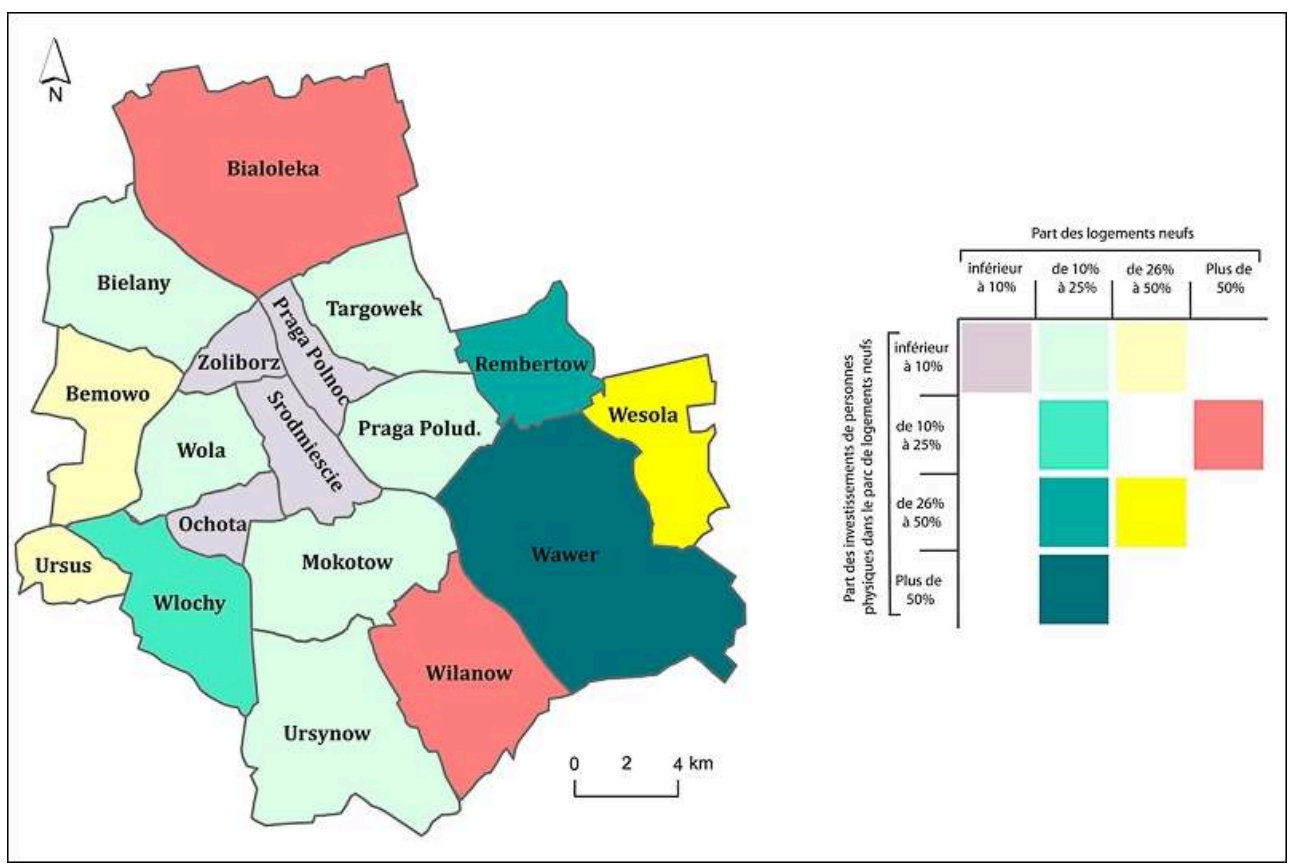

Source : GUS, 2012.

Ces nouvelles constructions posent en matière d'espace plusieurs problèmes. D'une part, des résidences fermées apparaissent, privatisant une partie des rues de la ville, et rendant encore plus visibles les inégalités socio-économiques qui émergent. D’autre part, le continuum urbain s'étale de plus en plus loin dans des quartiers périphériques où les infrastructures sociales, de transports ou commerciales sont moins développées, et où les collectivités locales interviennent très peu ou bien plus tard.

A titre d'exemple dans le quartier Wawer, les conflits spatiaux qu'engendrent ces constructions sont de l'ordre de l'occupation du sol lié à l'étalement urbain. Des promoteurs immobiliers ou des investisseurs individuels achètent des terrains agricoles ou forestiers à un prix plus faible que s'il s'agissait d'un terrain constructible. Après une demande de changement de statut d'occupation du terrain, rarement refusée, les investisseurs délimitent des parcelles plus petites qu'ils revendent ou construisent.

\section{Conclusion}

L'accession à la propriété privée dans les villes polonaises après 1989 a été complexe à mettre en place, car elle impliquait la réinsertion de la notion de propriété privée et la restructuration du secteur de l'habitat hérité de la période socialiste. À partir des années 1990, la voie la plus simple pour devenir propriétaire est l'achat d'un bien immobilier neuf. La majeure partie des constructions immobilières collectives ou individuelles est produite par et pour le secteur privé et s'adresse généralement aux individus ayant un statut économique plus élevé. Parallèlement, le secteur public a été peu à peu privatisé à partir de la moitié des années 1990, permettant à de nombreux Polonais de devenir propriétaires de leur logement communal ou coopératif. Grâce à cette politique particulière de privatisation, l'accession à la propriété a été 
théoriquement possible pour toutes les strates de la société sans être réservée à ceux dont le statut économique a progressé à partir des années 1990.

En revanche, ce processus a conduit à des situations hybrides de modes de propriété et à une multiplication des statuts de propriété à l'intérieur d'un même immeuble, rendant leur gestion extrêmement difficile. La restitution de certains biens immobiliers datant d'avant-guerre à leurs propriétaires d'origine (ou à leurs héritiers) a d'autant plus contribué à leur complexité. C'est pourquoi de nombreux conflits ont émergé entre anciens locataires et nouveaux propriétaires dont les intérêts divergent puisque les premiers cherchent à sauvegarder un loyer modéré, alors que les seconds souhaitent optimiser leur investissement.

De fait, en Pologne au terme de sa transition des modes d'accession à la propriété sont rendus possibles dans le secteur de l'habitat. De la même façon, l'hybridité de certaines structures dans lesquelles le statut des individus se situe entre celui de propriétaire et celui de locataire, comme la propriété coopérative, a tendance à s'atténuer au fil des années, se transformant généralement en propriété privée à part entière. Les politiques de privatisation de l'habitat ont ainsi porté leurs fruits. Toutefois, même si l'accession à la propriété a été possible pour de nombreux ménages de différents statuts socioéconomiques, il n'en reste pas moins qu'aucune politique envers le logement pour les plus défavorisés n'a été au cœur des initiatives de ces dernières années. À cet égard, les inégalités en termes de logements en Pologne n'ont cessé d'augmenter et l'accession à la propriété ne sera bientôt plus possible pour les moins aisés.

\section{BIBLIOGRAPHY}

Andrzejewski A., 1977. Sytuacja mieszkaniowa w Polsce w latach 1918-1974. [La situation du logement en Pologne de 1918 à 1974]. Warszawa, Państwowe Wydawnictwo Ekonomiczne, 447 p.

Bouloc C., 2013. Les élites dans les villes polonaises. Étude de Géographie sociale. Thèse sous la direction de P. Petsimeris et G. WĘcławowicz, University of Paris 1, Institute of Geography and Spatial Organization Polish Academy of Sciences, $376 \mathrm{p}$.

Carter F., Jordan P., Rey V. (dir.), 1998. Central Europe after the fall of the Iron Curtain - Geopolitical perspectives, spatial patterns and trends. Coll. "Wiener Osteuropa-Studien”, Peter Lang, Europäischer Verlag des Wissenschaften, Francfort/Berlin/New York/Paris/Vienne, 341 p.

Celińska-Janowicz D., 2010. Druga młodość czy upadek? Warszawskie osiedle Służew nad DolinkĄ $\mathrm{w}$ okresie transformacji. [La seconde jeunesse ou le déclin? La résidence varsovienne Służew nad DolinkĄ durant la transition]. Studia Regionalne i Lokalne, 1 (39), p. 89-104.

Cesarski M., 2007. Sytuacja mieszkaniowa w Polsce w latach 1988-2005. Varsovie, SGH, 375 p.

Cesarski M., 2010. Dorobek materialny spółdzielczości mieszkaniowej w Polsce 1956-2010.

[Artefacts des coopératives d'habitation en Pologne 1956-2010]. Communication au colloque à l'occasion des 120 ans de la création des coopératives d'habitation, Bydgoszcz, 4 novembre 2010. 
Coudroy de Lille L., 2009. La construction de logements à Varsovie depuis 1990 : une reprise en demi-teinte. In Berard E., Jaquant C. (dir.), Berlin-Varsovie-Moscou, L'architecture au-delà du mur. Picard, Paris, p. 203-220.

Coudroy de Lille L., 2012. L'habitat du « post » en Europe centrale et orientale. Historiens et géographes, $n^{\circ}$ 419, Editeur APHG, Paris, dossier p. 75-80.

GałĄzka A., 1998. Sytuacja mieszkaniowa ludności aglomeracji warszawskiej w latach 1970-1988. Zróżnicowania przestrzenne i tendencje zmian. [Situation d'habitation de la population dans l'agglomération varsovienne entre 1970 et 1988. Différentiations spatiales et tendances du changement]. Wydawnictwo Continuo, Prace Geograficzne nr 169, Wrocław, p. 154.

Gaudray L., 1995. L'habitat à Varsovie: un héritage et ses mutations. Thèse sous la direction d'A. Vant, Atelier national de Reproduction des Thèses, Lille, $471 \mathrm{p}$.

Glock B., 2007. Social and Spatial Consequences of the Restitution of Real Estate. In Stanilov K. (dir.), The Post-Socialist City, Urban Form and Space Transformations in Central Europe after Socialism. The Netherlands, Springer, p. 191-214.

Górczyńska M., 2012a. Zmiany zróżnicowań społecznych i przestrzennych w wybranych dzielnicach Warszawy i aglomeracji paryskiej: dynamika i aktorzy. [Changement de la différenciation sociospatiale des quartiers à Varsovie et dans l'agglomération parisienne: dynamiques et acteurs]. Thèse sous la direction de G. WĘcławowicz et M. Berger, University of Paris 1, Institute of Geography and Spatial Organization Polish Academy of Sciences, 291 p.

Górczyńska M., 2012b. Specificity of gated neighbourhoods in the Bielany district (Warsaw), Articulo - Journal of Urban Research [Online], 8 | 2012, Online since 29 June 2012, URL: http:// articulo.revues.org/2022

Górczyńska M., 2013. Changes in the social composition in pre-war housing in Warsaw. Communication au colloque AAG Annual Meeting, Los Angeles, 9-13 avril 2013.

Górczyńska M., 2014a. Enjeux entre acteurs publics et privés dans le secteur de l'habitat à Varsovie : le cas des immeubles revendiqués. In Belmessous F., Bonneval L., Coudroy de Lille L., Ortar L. (dir.), Logement et politique(s). Un couple encore d'actualité ? L'Harmattan, collection Habitat et société, p. 29-44.

Górczyńska M., 2014b. Towards social upgrading: conceptualization of social change in the postsocialist city (the case of Warsaw, Poland). Communication au colloque IGU, Cracovie, 18-22 août 2014.

Hegedüs J., Tosics I., 1998. Towards New Models of the Housing System. In Enyedi G. (dir.), Social Change and Urban Restructuring in Central Europe. Budapest, Akadémiai Kiado, p. 137-167.

Herbst I., Muzioł-WĘcławowicz A., 1993. Housing in Poland, Problems and reforms. Cities, August 1993, p. 246-256.

Kałkowski L., 2010. 20 years of Polish real estate market, monitoring for the period 1990-2009. Cracow.

Le Gall J., Rougé L., 2014. Oser les entre-deux! Carnets de géographes, $\mathrm{n}^{\circ} 7$, rubrique Carnets de débats, p. 1-23.

Marcuse P., 1996. Privatization and its Discontents: Property Rights in Land and Housing in the Transition in Eastern Europe. In Andrusz G., Harloe M., Szelenyi I., Cities after Socialism, Urban and Regional Change and Conflict in Post-socialist Societies. Blackwell Publishers, p. 286-317. 
Ministère des Transports et de la Construction, 2005. Strategia długofalowego rozwoju sektora mieszkaniowego na lata 2005-2025, Zał Ącznik: uwarunkowania sektora mieszkaniowego 2005-2013. [Stratégie à long terme du développement du secteur de l'habitat entre 2005 et 2025, annexe : les conditionnements du secteur de l'habitat 2005-2013]. Warszawa.

Rey V., 1990. Feu l'Europe de l'Est. Annales de Géographie, t. 99, n555, p. 564-578.

WĘcławowicz G., 1998. Social Polarisation in Postsocialist Cities: Budapest, Prague and Warsaw. In Enyedi G. (dir.) Social Change and Urban Restructuring in Central Europe. Budapest, Akadémiai Kiadó, p. 55-66.

WĘcławowicz G., Gaudray-Coudroy, L., 1998. Le renouveau du secteur privé et la recomposition de l'espace social à Varsovie. In Bertrand, J-R., Chevalier, J. (dir.), Logement et habitat dans les villes européennes. Paris, L'Harmattan, p. 59-78.

Zaniewska H. (dir.), 2005. Mieszkania dla ubogich w zrównoważonym rozwoju miast [Le logement pour les pauvres et le développement durable des villes]. Kraków, Instytut Rozwoju Miast, 102 p.

Zaniewska H. (dir.), Uchman B., Urbańska W., DĄbkowski N., 2013. Informacje o mieszkalnictwie, wyniki monitoringu za 2012 rok. Kraków, Instytut Rozwoju Miast, p. 92.

Zborowski A., 2005. Przemiany struktury społeczno-przestrzennej regionu miejskiego w okresie realnego socjalizmu i transformacji ustrojowej (na przykładzie Krakowa). [Changements de la structure sociospatiale de l'espace urbain durant le socialisme réel et la transformation du régime (le cas de Cracovie)]. Kraków, Instytut Geografii i Gospodarki Przestrzennej Uniwersytetu Jagiellońskiego, 576 p.

\section{NOTES}

1. Les lois précédentes n'utilisent pas la notion de "propriété coopérative ». Par exemple, la loi de coopérative de 1961 distinguait trois types de coopératives (C) : les C d'habitation, les C d'habitation-construction ou les $\mathrm{C}$ de maisons individuelles. Les deux premiers types se distinguaient quant à leurs définitions de la notion de location coopérative. En effet, les locataires des C d'habitation-construction disposaient de plus de droits que les locataires des C d'habitation (par exemple, il était possible d'hériter du logement). Ainsi, tous les éléments définissant les locations coopératives dans les $C$ d'habitation-construction font penser à une propriété coopérative, mais le terme n'est pas utilisé. Par ailleurs, la situation des $C$ de maisons individuelles était spécifique, car les coopératives étaient créées seulement pour la construction des maisons. Dès lors que celles-ci étaient achevées, la propriété des maisons était transmise aux membres de la coopérative. Ainsi, ce n'est que dans le texte de la loi coopérative de 1982 que le terme de " propriété coopérative » (pl. mieszkanie spółdzielcze na prawie własnościowym) apparaît de façon formelle pour les $\mathrm{C}$ d'habitation.

2. Towarzystwa Budownictwa Społecznego (TBS).

3. Les données statistiques proviennent de deux sources : Real Estate turnover in 2009, Statistical Information and Elaborations, GUS, Warsaw, 2010 et Kałkowski, 2010.

4. 59186 transactions en 1990 pour 252234 en 2006.

5. En Pologne, il existe plusieurs possibilités de financer l'acquisition d'un logement par un prêt bancaire dans d'autres monnaies (euros, francs suisse). Avant la crise de 2008 et avant l'augmentation de la valeur de franc suisse, beaucoup de ménages polonais choisissaient principalement cette monnaie pour souscrire à des crédits immobiliers.

6. Les trois premières formes d'occupation de logements sont statistiquement comptabilisées comme des logements coopératifs alors que la quatrième forme est assimilée à la propriété privée, bien que physiquement ces logements se situent dans des immeubles coopératifs. 
7. Sources : données statistiques de la Mairie de Varsovie, 2014.

8. En polonais « Komitet Obrony Lokatorów », site internet : http://lokatorzy.info.pl/o-nas/

\section{ABSTRACTS}

The reintroduction of the concept of private property and the establishment of a real estate market in Poland since 1989, have permanently changed the housing sector. The homeownership was restored; however, its implementation has not been simple and resulted in the creation of complex forms of ownership. This paper aims at explaining in detail how the housing sector has been restructured, shedding the light on the different ownership status that have multiplied in the recent years. Additionally, it analyses contemporary social and spatial conflicts provoked by changes in the ownership.

La réintroduction de la notion de propriété privée et la mise en place d'un marché de l'immobilier libéral en Pologne après 1989 a durablement bouleversé le secteur de l'habitat. De nouveau rendue possible, l'accession à la propriété n'a pourtant pas été évidente à mettre en place et les statuts de propriété sont encore aujourd'hui extrêmement complexes. Cet article a pour but d'expliquer en détail la manière dont le secteur de l'habitat s'est restructuré, mais aussi de rendre plus lisibles les statuts de propriété qui se sont multipliés ces dernières années ainsi que d'analyser les conflits sociaux et spatiaux qui résultent de l'accession à la propriété aujourd'hui.

\section{INDEX}

Keywords: Ownership, housing, private property, post-socialist, Poland, Warsaw Mots-clés: Accession à la propriété, logement, propriété privée, post-socialiste, Pologne, Varsovie

\section{AUTHORS}

\section{CAROLINE BOULOC}

Caroline Bouloc, c.bouloc@parisgeo.cnrs.fr, docteure en Géographie, est chercheure associée au CNRS, UMR 8504 Géographie-cités. Elle a publié récemment :

- Bouloc C., 2012. Miejsce zamieszkania elity w Warszawie, wybory i aspiracje. In Paradowska E. (dir.), Procesy Gentryfikacji kontra Procesy wykluczenia społecznego w Polskich Miastach, XXV Konversatorium Wiedzy o Mieście, [Lieux d'habitation des élites à Varsovie: choix et aspirations. In Paradowska E. (dir.), Processus de gentrification contre processus d'enfermement social dans les villes polonaises]. p. 257-267.

- Bouloc C., 2011. Urban Elites in Polish intra-metropolitan space: the case of Warsaw, Krakow and Lodz. In Kalogirou S. (ed.), ecQTG $2011-17^{\text {th }}$ European Colloquium on Quantitative and Theorical Geography - Proceedings, 2-5 September 2011, Greek Society for Demographic Studies, Harokopio University, Geography department, ID 33, p. $42-51$. 


\section{MAGDALENA GÓRCZYŃSKA}

Magdalena Górczyńska, mgor@twarda.pan.pl, docteure en Géographie, est chargée de recherche à l'Institut de Géographie et d'Aménagement du Territoire (Académie des Sciences de Pologne) et chercheure invitée au CNRS, UMR 8504 Géographie-cités (2013-2016). Elle a publié récemment :

- Górczyńska M., 2014. Enjeux entre acteurs publics et privés dans le secteur de l'habitat à Varsovie : le cas des immeubles revendiqués. In Belmessous F., Bonneval L., Coudroy de Lille L., Ortar L. (dir.), Logement et politique(s). Un couple encore d'actualité ? L'Harmattan, collection Habitat et société, p. 29-44.

- Górczyńska M., 2014. Unique or universal? Mechanisms and processes of social change in post-socialist Warsaw, Hungarian Geographical Bulletin, 63(3), pp.255-277, DOI: 10.15201/hungeobull.63.3.2, http:// www.mtafki.hu/konyvtar/kiadv/HunGeoBull2014/HunGeoBull_63_3_2.pdf

- Górczyńska M., 2012. Specificity of gated neighbourhoods in the Bielany district (Warsaw), Articulo - Journal of Urban Research [Online], 8 | 2012, Online since 29 June 2012, URL: http:// articulo.revues.org/2022 